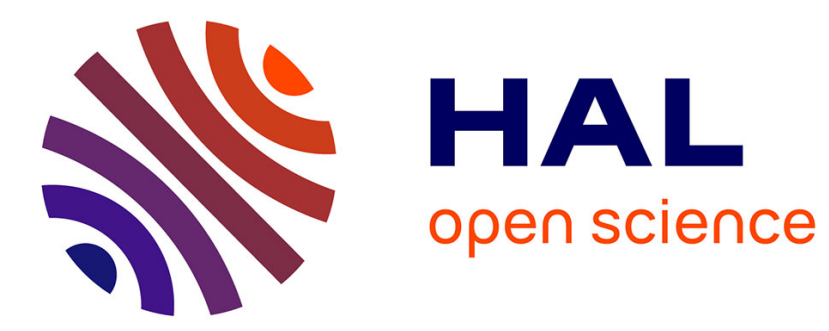

\title{
Breakdown of the electron-spin motion upon reflection at metal-organic or metal-carbon interfaces. II.
}

\author{
M. Ochapski, E. Urbain, F. Djeghloul, V. Speisser, H. Majjad, D. Spor, A. D.
} Vu, Johann Coraux, N. Rougemaille, G. Chen, et al.

\section{- To cite this version:}

M. Ochapski, E. Urbain, F. Djeghloul, V. Speisser, H. Majjad, et al.. Breakdown of the electron-spin motion upon reflection at metal-organic or metal-carbon interfaces. II.. Physical Review B, 2016, 93 (17), pp.174411. 10.1103/PhysRevB.93.174411 . hal-01317093

\section{HAL Id: hal-01317093 \\ https://hal.science/hal-01317093}

Submitted on 1 Sep 2021

HAL is a multi-disciplinary open access archive for the deposit and dissemination of scientific research documents, whether they are published or not. The documents may come from teaching and research institutions in France or abroad, or from public or private research centers.
L'archive ouverte pluridisciplinaire HAL, est destinée au dépôt et à la diffusion de documents scientifiques de niveau recherche, publiés ou non, émanant des établissements d'enseignement et de recherche français ou étrangers, des laboratoires publics ou privés. 


\title{
Breakdown of the electron-spin motion upon reflection at metal-organic or metal-carbon interfaces. II.
}

\author{
M. Ochapski, ${ }^{1}$ E. Urbain, ${ }^{1}$ F. Djeghloul,,${ }^{1,}$ V. Speisser, ${ }^{1}$ H. Majjad, ${ }^{1}$ D. Spor, ${ }^{1}$ A. D. Vu, ${ }^{2,3}$ J. Coraux, ${ }^{2,3}$ N. Rougemaille, ${ }^{2,3}$ \\ G. Chen, ${ }^{4}$ A. K. Schmid, ${ }^{4}$ M. Suzuki, ${ }^{5}$ T. Yasue, ${ }^{5}$ T. Koshikawa, ${ }^{5}$ H. Bulou, ${ }^{1}$ and W. Weber ${ }^{1}$ \\ ${ }^{1}$ Institut de Physique et Chimie des Matériaux de Strasbourg, UMR 7504, CNRS-UdS, 23 rue du Loess, BP 43, \\ 67034 Strasbourg Cedex 2, France \\ ${ }^{2}$ CNRS, Institut NEEL, F-38000 Grenoble, France \\ ${ }^{3}$ Université Grenoble Alpes, Institut NEEL, F-38000 Grenoble, France \\ ${ }^{4}$ NCEM, Molecular Foundry, Lawrence Berkeley National Laboratory, Berkeley, California 94720, USA \\ ${ }^{5}$ Fundamental Electronics Research Institute, Osaka Electro-Communication University, Neyagawa, Osaka 572-8530, Japan
}

(Received 18 April 2016; published 17 May 2016)

\begin{abstract}
A breakdown of the spin dependence of the electron reflection due to organic molecules or amorphous carbon deposited onto a metallic film has been observed in the past. The goal of the present work is to further elucidate the physics of this phenomenon by studying it in ways not yet studied in the past. The most intriguing observation of the present study is that the breakdown phenomenon appears in a relatively well defined electron energy range between 2 and about $200 \mathrm{eV}$ kinetic energy. Outside this energy range the breakdown phenomenon is not observed. However, an explanation of the breakdown phenomenon is still missing.
\end{abstract}

DOI: 10.1103/PhysRevB.93.174411

\section{INTRODUCTION}

When an electron beam is reflected from a surface, the reflection properties, including both reflectivity and reflection phase, depend in general on the orientation of the spin polarization vector of the incident beam. There are two main physical phenomena giving rise to spin-dependent electron reflection: exchange and spin-orbit interaction. While exchange interaction is essentially an outcome of the Pauli principle, requiring the total wave function of the electrons to be antisymmetric with respect to a permutation of the electrons, spin-orbit interaction, being most pronounced in heavy materials, is due to the interaction of the spin of an electron with its own angular momentum in the electric field of a strong scattering potential. In fact, both in ferromagnetic materials such as Fe or Ni (exchange-governed systems) and in heavy materials such as $\mathrm{W}$ or $\mathrm{Au}$ (spin-orbit governed systems) a spin-dependent electron reflection at the surfaces of these materials is observed [1].

However, some of us discovered very recently a completely unexpected behavior of the spin-dependent electron reflection when a ferromagnetic surface is covered by submonolayer amounts of organic molecules [2] (Ref. [2] will be called "paper I" in the following). In fact, the deposition of submonolayer amounts of nonmagnetic organic molecules makes the reflection of electrons independent of the spin, i.e., both the reflectivity and the reflection phase become independent of the spin of the incident electrons (see Fig. 1). Such a spinindependent reflection is only expected for film thicknesses (of any nonmagnetic material) larger than the inelastic mean free path of the electrons of several monolayers (ML), but not for such low coverage as studied in paper I and this work. This phenomenon, which we call breakdown phenomenon (BP), seems to be very general as it is in particular independent both

\footnotetext{
*Present address: Université de Ferhat Abbas Sétif 1, Faculté de Technologie, Sétif, Algeria.
}

of the metallic substrate and the organic molecule [2]. The BP appears in experiments with ferromagnetic systems, as well as in experiments with heavy nonmagnetic materials in which only spin-orbit interaction can lead to a spin dependence of the reflected intensity [2]. Moreover, we showed that not only organic molecules lead to the BP but also the presence of amorphous carbon (a-C) [2]. This suggests that carbon atoms play a dominant role in the BP. We emphasize that no other element or compound exhibiting a similar behavior is known at the moment, and seems therefore indicative of a completely novel property of the interaction of spin-polarized electrons with carbon and carbon-based systems. We emphasize that the $\mathrm{BP}$ cannot be explained in terms of well known concepts such as [2]

(1) demagnetization (i.e., no magnetic dead layers at the ferromagnetic metal-organic interface),

(2) breakdown of the magnetic signal due to changes of remanence, coercive field, anisotropy etc.,

(3) depolarization of the spin-polarized electron beam (i.e., no spin-flip scattering),

(4) hybridization between molecules and substrate (i.e., the presence of the BP is independent of the particular electronic structure of both the organic molecules and the substrate),

(5) quantum-well states within the molecular layer,

(6) coherence effects (i.e., the BP appears also with incoherent electrons), and

(7) interfacial dipole effects.

We note, in particular, that the observation of the BP is not at all in contradiction to our recent spin-resolved photoemission experiments $[3,4]$, in which highly spin-polarized interface states are observed in ferromagnetic metal-organic and metalcarbon systems. In fact, the BP is only revealed in experiments in which the behavior of the spin-dependent electron reflection is studied, which is neither the case in photoemission spectroscopy nor in many other experimental methods which have been used in the past to study ferromagnetic metal-organic interfaces. Thus, it does not come as a surprise that the BP is not observed by other experimental methods. The goal of 


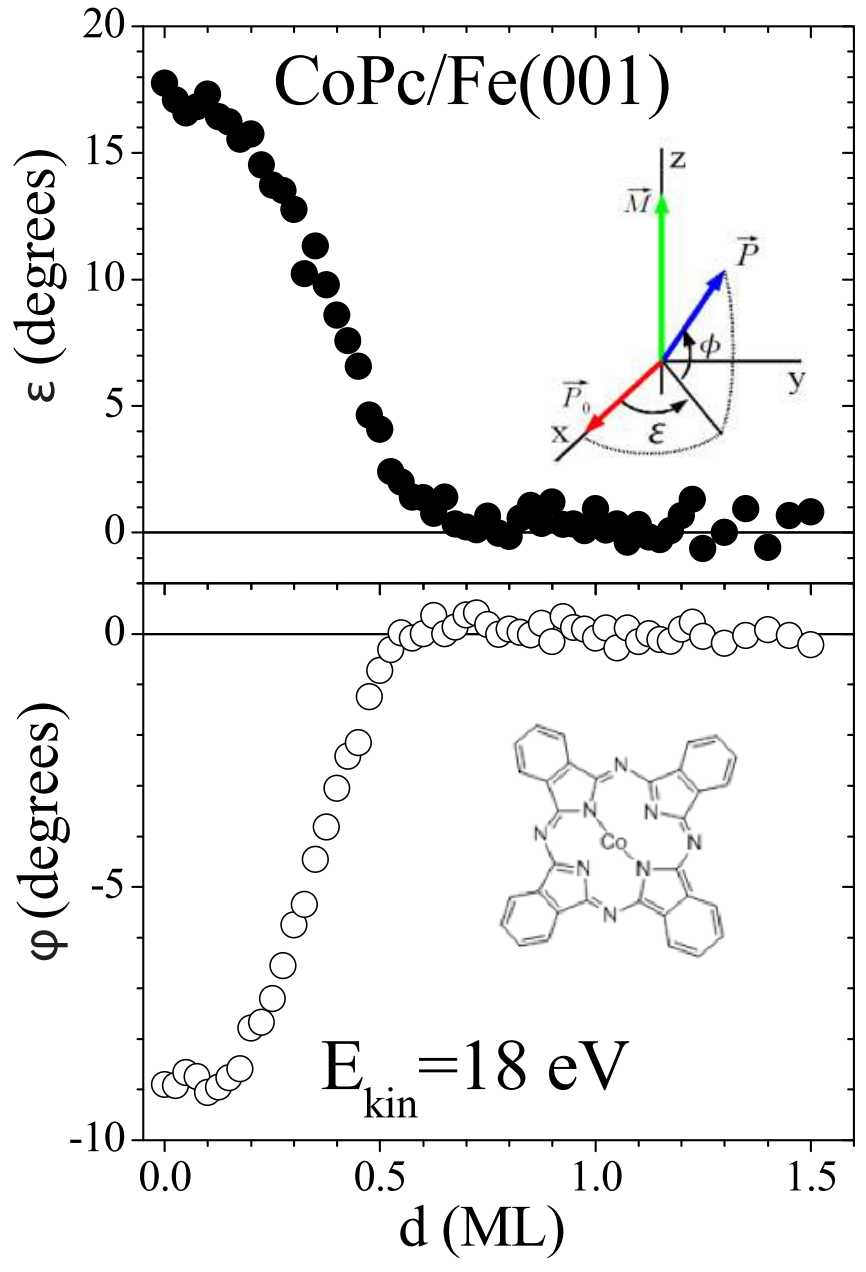

FIG. 1. For the system Co-phthalocyanine $(\mathrm{CoPc}) / \mathrm{Fe}(001)$ the precession angle $\varepsilon$ (top) and the rotation angle $\phi$ (bottom) of reflected electrons are shown as a function of the Pc thickness in monolayers (ML). The top inset shows the two types of motion of the spin-polarization vector. If the initial spin polarization $\mathbf{P}_{0}$ is oriented perpendicularly with respect to the magnetization $\mathbf{M}$ of the ferromagnet the spin polarization precesses around $\mathbf{M}$ by an angle $\varepsilon$ and rotates simultaneously in the plane $\mathbf{P}-\mathbf{M}$ by an angle $\phi$. The precession angle is the difference of the spin-dependent reflection phases and the rotation angle is determined by the difference of the spin-dependent reflectivities [2,5]. Consequently, electron reflection becomes independent of the spin when both quantities disappear which is the case for Pc thicknesses already well below 1 ML. The bottom inset shows a CoPc molecule. The kinetic energy of the primary electrons is $18 \mathrm{eV}$.

the present paper is to further elucidate the physics of this phenomenon by studying it in ways not yet done in paper I. However, an explanation of the BP is still missing.

\section{SPIN-DEPENDENT REFLECTION AT SURFACES}

The changes in the spin-dependent reflectivity and reflection phase as a function of the organic layer thickness can most easily be studied if the spin polarization vector $\mathbf{P}_{0}$ of the incident electrons is chosen perpendicularly with respect to the magnetization direction $\mathbf{M}$ in exchange-governed systems or to the normal vector $\mathbf{n}$ [6] of the reflection plane in spin-orbit governed systems. Supposing for simplicity a completely polarized electron beam, the spin part of the incident electron wave function is in this case a coherent superposition of a spin-up (magnetic moment parallel to $\mathbf{M}$ or to $\mathbf{n}$ ) and a spin-down (magnetic moment antiparallel to $\mathbf{M}$ or to $\mathbf{n}$ ) wave function having equal amplitudes, i.e., both the reflectivities and the reflection phases are identical. Because of the spindependent reflection at the surface, the wave functions for the two spin components are different. This change of the spin wave function corresponds in real space to a spin motion, comprising a precession by an angle $\varepsilon$ of the spin polarization vector around $\mathbf{M}$ or $\mathbf{n}$ (due to the spin-dependent reflection phase) and a rotation in the plane $\mathbf{P}-\mathbf{M}$ or $\mathbf{P}-\mathbf{n}$ by an angle $\phi$ (due to the spin-dependent reflectivity) (see top inset in Fig. 1 for the exchange-governed case). We emphasize that the spindependent reflectivity can also be studied by measurements in which $\mathbf{P}_{0}$ is parallel or antiparallel to $\mathbf{M}$ or $\mathbf{n}$. In this case a reflectivity or intensity asymmetry $A$ can be measured which is directly related to the rotation angle $\phi$ in a spin-motion experiment [5]. The precession angle $\varepsilon$, however, cannot be determined within such an experiment.

For more details about the theory of electron-spin motion, the setup of the spin-motion experiment and certain details of the sample preparation we refer the reader to paper I. Only those aspects which have not yet been presented in paper I, for instance the principle of spin-polarized low-energy electron microscopy (SPLEEM), will be mentioned in the present paper.

\section{RESULTS AND DISCUSSION}

\section{A. BP for spin-orbit governed systems}

In paper I it has already been shown that the BP is not only limited to exchange-governed systems, i.e., systems in which the spin dependence of the electron reflection is mainly induced by ferromagnetism, but exists also in spin-orbit governed systems, i.e., systems in which the spin dependence of the electron reflection is dominated by spin-orbit interaction. In these experiments the initial spin polarization vector $\mathbf{P}_{\mathbf{0}}$ is oriented perpendicularly with respect to the reflection plane. By measuring the reflected electron intensity $R$ in two spin configurations, namely with $\mathbf{P}_{\mathbf{0}}$ parallel ( $\left.\uparrow \uparrow\right)$ and antiparallel $(\uparrow \downarrow)$ to the normal $\mathbf{n}$ of the reflection plane we obtain the so-called spin-orbit asymmetry $A_{\text {so }}$ (normalized to the value of $\left.P_{0}\right)$ :

$$
A_{\mathrm{so}}=\frac{1}{P_{0}} \frac{R\left(\mathbf{P}_{\mathbf{0}} \uparrow \uparrow \mathbf{n}\right)-R\left(\mathbf{P}_{\mathbf{0}} \uparrow \downarrow \mathbf{n}\right)}{R\left(\mathbf{P}_{\mathbf{0}} \uparrow \uparrow \mathbf{n}\right)+R\left(\mathbf{P}_{\mathbf{0}} \uparrow \downarrow \mathbf{n}\right)} .
$$

Interestingly, this spin-orbit induced quantity behaves as a function of the organic layer thickness similarly to the exchange asymmetry $A_{\mathrm{ex}}$ and other exchange-governed quantities such as the spin precession and rotation angles $\varepsilon$ and $\phi$ in systems in which organic molecules or a-C are deposited onto ferromagnetic surfaces (see paper I). Importantly, Fig. 2, which shows $A_{\text {so }}$ as a function of the kinetic electron energy for different $\mathrm{H}_{2} \mathrm{Pc}$ coverages on $\mathrm{Pt}$ from 0 to $1.12 \mathrm{ML}$, proves that the BP is not limited to the few particular energies studied in paper I but that it is present at least within the energy range from 13 to $35 \mathrm{eV}$. 


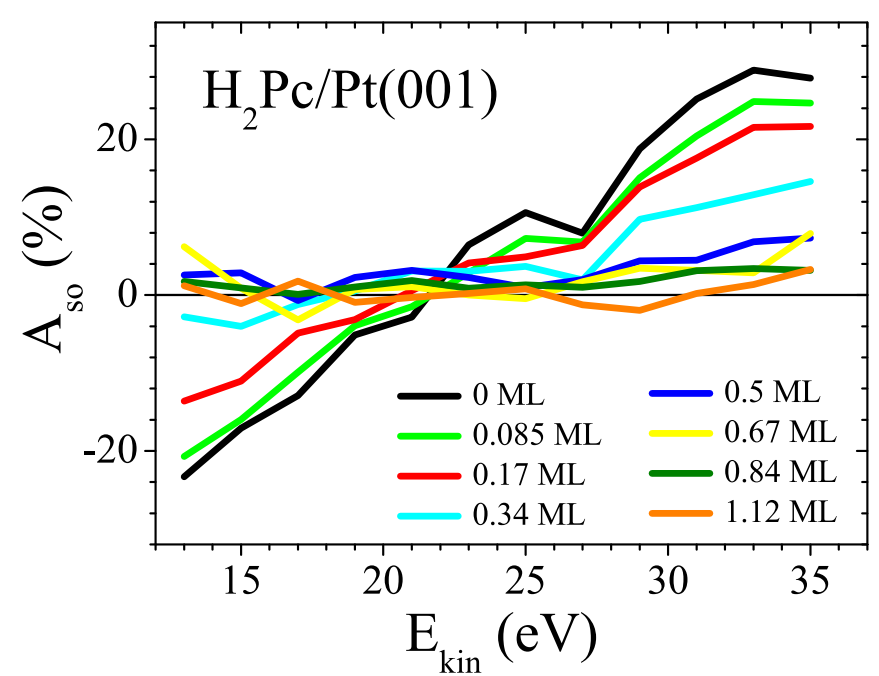

FIG. 2. $A_{\text {so }}$ of $\mathrm{Pt}(001)$ covered by different thicknesses of $\mathrm{H}_{2} \mathrm{Pc}$ as a function of the kinetic electron energy.

To check whether the spin-orbit induced spin precession around the normal $\mathbf{n}$ of the reflection plane is also subject to the BP $\mathbf{P}_{\mathbf{0}}$ has been oriented perpendicularly with respect to $\mathbf{n}$. We note that the electron-spin motion in spin-orbit governed systems such as Pt has been treated in detail by Kessler [7].

The spin-orbit induced spin precession $\varepsilon_{\text {so }}$ is shown as a function of $\mathrm{H}_{2} \mathrm{Pc}$ coverage in Fig. 3 for electrons of $7 \mathrm{eV}$ kinetic energy. The BP is observed with the same abrupt decrease as seen for $A_{\mathrm{so}}$. Our results thus show that the spin-orbit induced electron-spin precession behaves similarly to the exchange-induced spin precession in ferromagnetic systems. Consequently, not only the spin-dependent reflectivities (entering the expression for $A_{\mathrm{so}}$ ) are subject to the $\mathrm{BP}$ but also the spin-dependent reflection phases (entering the expression for the spin precession). By summarizing the observations presented here and in paper I we can state that all quantities, be it in an exchange-governed or in a spin-orbit

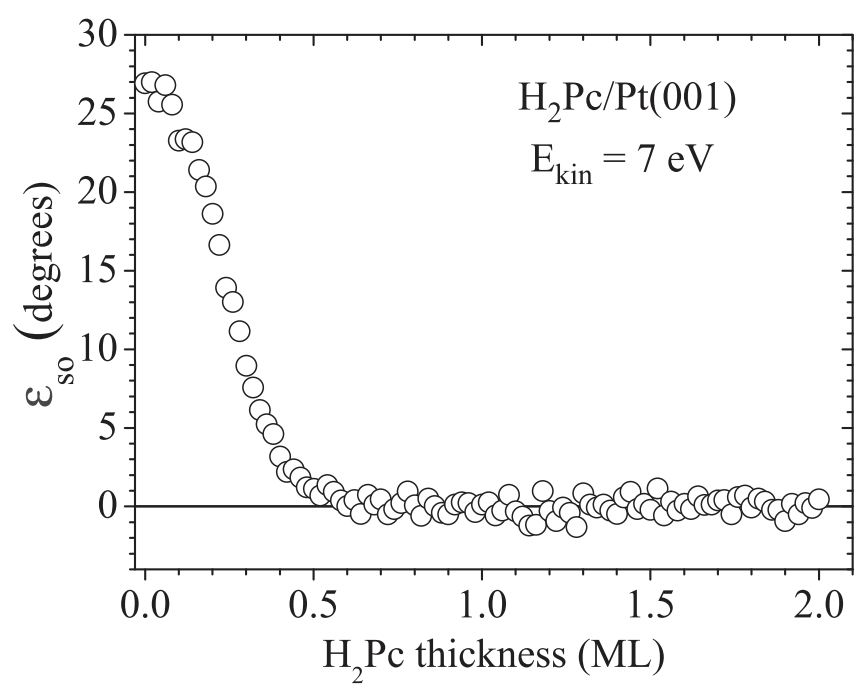

FIG. 3. The spin-orbit induced spin precession $\varepsilon_{\text {so }}$ as a function of $\mathrm{H}_{2} \mathrm{Pc}$ on $\mathrm{Pt}(001)$. The kinetic energy of the incident electrons is $7 \mathrm{eV}$.

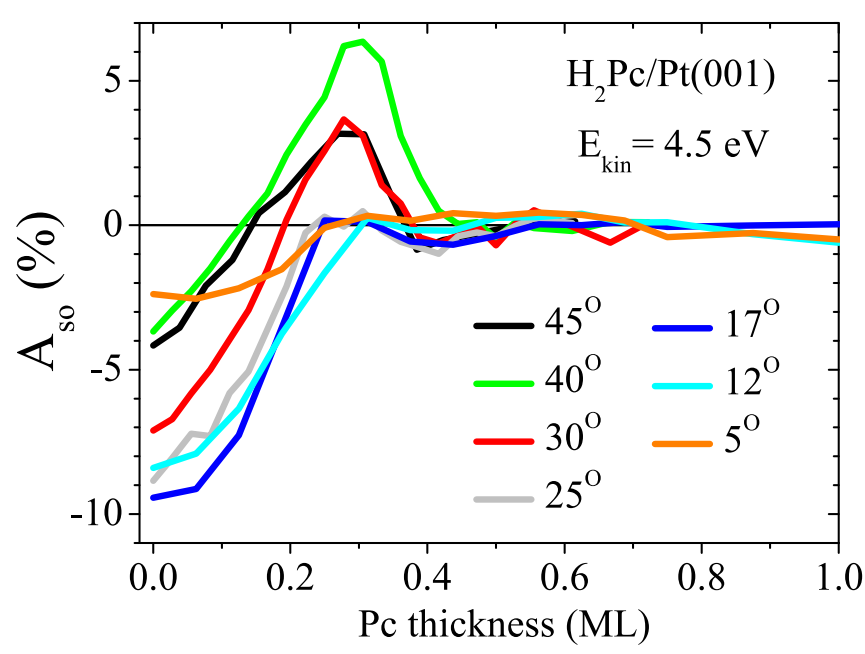

FIG. 4. $A_{\mathrm{so}}$ of $\mathrm{Pt}(001)$ as a function of $\mathrm{H}_{2} \mathrm{Pc}$ thickness for different incidence angles of the electrons. The kinetic energy of the electrons is $4.5 \mathrm{eV}$.

governed system, which depend either on the spin-dependent electron reflectivities or the spin-dependent reflection phases are without exception subject to the BP.

The results obtained concerning spin-orbit governed systems raise an interesting question, namely whether the BP seen with electrons might also be present in experiments with positrons. We note that, though exchange effects and thus any exchange asymmetry is not expected for positrons (there is no exchange interaction between the incident positrons and the electrons within the sample) a spin-orbit asymmetry is expected to appear with positrons [8].

\section{B. Does the presence of the BP depend on the angle of incidence of the electrons?}

The experimental apparatus is set up to realize a scattering geometry with $45^{\circ}$ incidence angle. Thus, all measurements presented in paper I have been performed with this particular incidence angle of the electrons. To verify that the BP is not limited to a narrow incidence angle range just around $45^{\circ}$ we had to undertake a study of the $\mathrm{BP}$ as a function of the incidence angle. For this, however, the experimental conditions had to be modified by applying a magnetic field perpendicularly to the reflection plane of the electrons such that the electrons follow a curved trajectory and arrive on the sample under an angle different from $45^{\circ}$. In this way a large range of incidence angles could be realized.

In the following we present measurements we have performed with the spin-orbit governed system Pt. Figure 4 shows $A_{\mathrm{so}}$ of $\mathrm{Pt}(001)$ at a kinetic energy of $4.5 \mathrm{eV}$ as a function of $\mathrm{H}_{2} \mathrm{Pc}$ thickness for different incidence angles up to $45^{\circ}$. The essential message from these measurements is that the $\mathrm{BP}$ is present in a wide range of incidence angles and not limited just to $45^{\circ}$. As a function of the incidence angle we see the development of an additional structure which leads to a clear sign change. However, we should mention that similar curves with more complexity have also been observed in ferromagnetic systems for certain configurations (energy, 


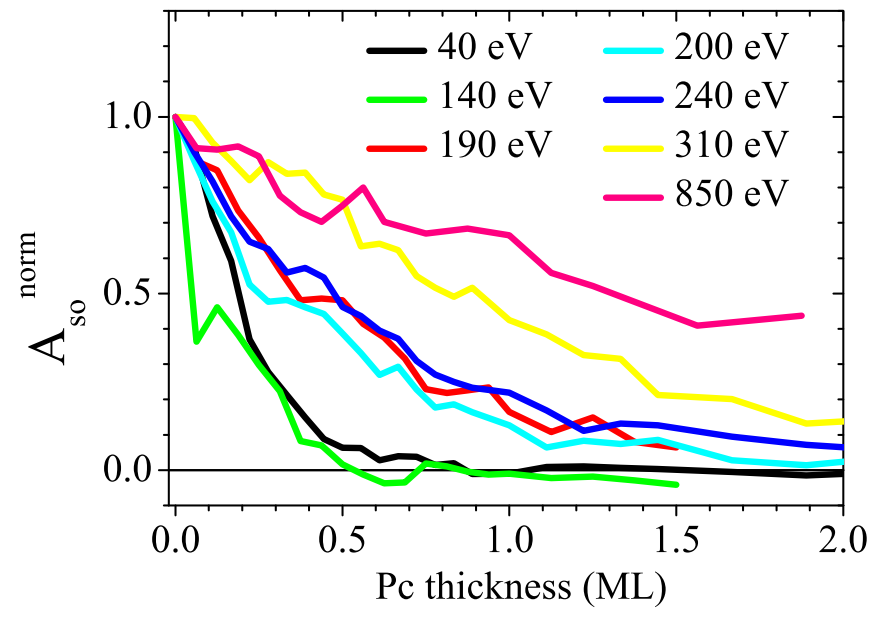

FIG. 5. The normalized spin-orbit asymmetry $A_{\mathrm{so}}^{\text {norm }}$ of Pt as a function of $\mathrm{H}_{2} \mathrm{Pc}$ thickness for different kinetic energies of the incident electrons.

organic layer, substrate, etc.; see for instance the behavior of $\phi$ as a function of Pc thickness in Fig. 7 of paper I).

We can thus conclude that the orientation of the electron wave vector $\mathbf{k}$ is of no importance for the appearance of the BP. We note in particular that experiments in normal incidence geometry (not shown) prove that electrons without a wave vector component parallel to the sample surface are subject to the BP. The same is also true for electrons possessing (almost) no wave vector component perpendicular to the surface. Experiments (not shown) performed in grazing incidence reveal the same type of BP. We note that we have already shown in paper I that the presence of the BP is also independent of the orientation of the electrons' spin polarization vector. Consequently, neither the orientation of the wave vector nor of the spin polarization vector will enter in any explanation of the BP.

\section{How does the BP depend on the electron energy?}

In the study of exchange-governed systems reported in paper I we found that the BP is present in the electron kinetic energy range from 3 to $130 \mathrm{eV}$. For experimental reasons we could not go to lower energies in our spin-motion setup. On the other hand, the upper limit of the energy is determined by the fact that the exchange interaction and thus the exchange asymmetry as well as related quantities such as the precession and the rotation angle become vanishingly small at higher energies. Thus, there is no signal anymore which can be followed as a function of molecular coverage. However, this situation is different in the case of spin-orbit governed systems for which the spin-orbit asymmetry $A_{\mathrm{so}}$ can exhibit large values even at very high energies. In the following we will show how $A_{\text {so }}$ behaves as a function of Pc coverage at much higher kinetic energies.

The results of the experiments within a wider energy range are presented in Fig. 5. For a better comparison of the data for different energies the normalized spin-orbit asymmetry $A_{\mathrm{so}}^{\text {norm }}=A_{\mathrm{so}} / A_{\mathrm{so}}(d=0)$ is shown.

While for kinetic energies of 40 and $140 \mathrm{eV}$ the $\mathrm{BP}$ is fully present, it disappears gradually for larger energies and

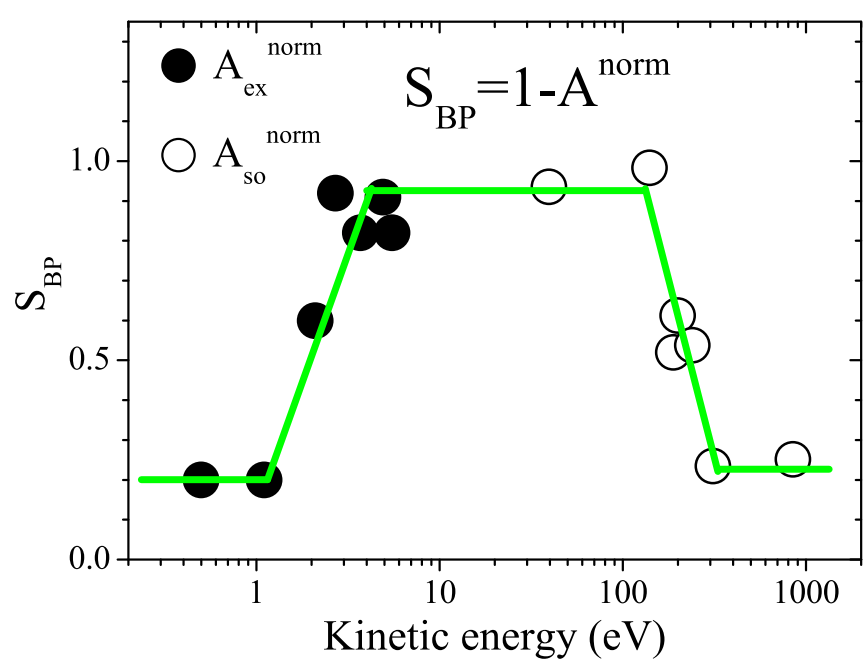

FIG. 6. The strength of the BP $S_{\mathrm{BP}}$ as a function of the kinetic electron energy of the incident electrons. Open symbols: $S_{\mathrm{BP}}$ derived from $A_{\mathrm{so}}$ measurements, full symbols: $S_{\mathrm{BP}}$ derived from $A_{\mathrm{ex}}$ measurements. The line is a guide to the eye. Note the logarithmic energy scale.

no indication of it is seen at $310 \mathrm{eV}$ or higher kinetic energies. We note that the overall decrease of the $A_{\mathrm{so}}$ signal as a function of Pc thickness which is extended over a much larger thickness range has nothing to do with the BP but is simply due to the finite inelastic mean free path (IMFP) of the electrons. The decrease in the slope with increasing energy at larger coverage is thus explained by the fact that the IMFP increases with increasing energy. In fact, fits of the data for 310 and $850 \mathrm{eV}$ kinetic energy with a decaying exponential result in IMFP values of about 1.2 and $2.3 \mathrm{~nm}$, respectively, which are close to those obtained by Tanuma et al. for organic compounds [9]. The data for the lowest kinetic energies, however, would give, if fitted by an exponential decay, unreasonably small IMFP values. This is not astonishing as the BP with its quite abrupt decay is present at these lower electron energies.

In order to see better how the "strength" of the BP $S_{\mathrm{BP}}$ varies with kinetic energy, we present in Fig. 6 a different plot, which shows $S_{\mathrm{BP}}=1-A_{\mathrm{so}}^{\text {norm }}$ obtained at a Pc thickness of $0.5 \mathrm{ML}$ for the different electron energies (see open symbols in Fig. 6). If $S_{\mathrm{BP}}$ is close to 1 the BP is considered to be complete, while a small value is considered to reflect the absence of the BP. We emphasize that the precise values of this quantity should be considered with caution, in particular, as the varying IMFP has not been taken into account. It is nevertheless a good indicator of the BP and we see that the BP disappears for kinetic electron energies above about $200 \mathrm{eV}$.

Is the disappearance of the $\mathrm{BP}$ at higher kinetic energies only a question of the time being spent by the electrons within the molecular layer? As the latter is proportional to $1 / \sqrt{E_{\text {kin }}}$ we should rather expect a more gradual linear-type decrease of $S_{\mathrm{BP}}$ with increasing energy instead of this relatively abrupt behavior. Moreover, we will see below that a non-BP behavior is also observed for very low kinetic energies.

In the following we will discuss our energy-dependent results we obtained by spin-polarized low-energy electron microscopy (SPLEEM) [10]. Figure 7 shows the normalized 


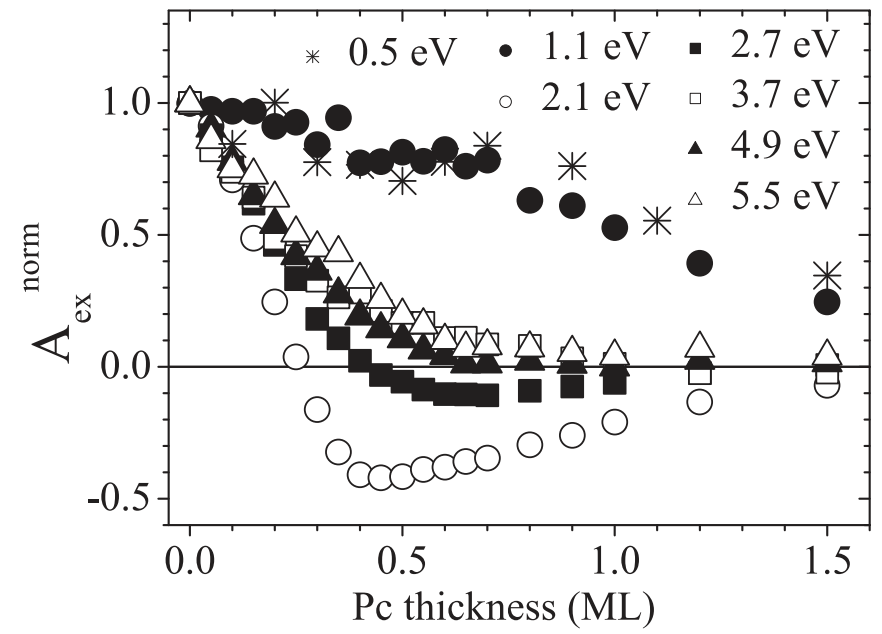

FIG. 7. The normalized exchange asymmetry $A_{\mathrm{ex}}^{\text {norm }}$ of $\mathrm{Co} / \mathrm{W}(110)$ as a function of $\mathrm{H}_{2} \mathrm{Pc}$ thickness for different kinetic energies of the incident electrons (see text).

exchange asymmetry $A_{\mathrm{ex}}^{\text {norm }}=A_{\mathrm{ex}} / A_{\mathrm{ex}}(d=0)$ obtained with SPLEEM as a function of $\mathrm{H}_{2} \mathrm{Pc}$ thickness on $\mathrm{Co} / \mathrm{W}(110)$ for different kinetic energies of the incident electrons. For details concerning the preparation of Co and Fe films on W(110) see Refs. [11,12] and Refs. [13,14], respectively. We note that the work function of the metallic substrate film changes as a function of Pc coverage (not shown). Consequently, the kinetic energy of the incident electrons with respect to the vacuum level of the sample varies as well as a function of Pc coverage. Up to now we mostly considered kinetic energy values which are much larger than the work function changes (up to about $-0.9 \mathrm{eV}$ in the case of Pc coverage). For this reason we did not take the thickness-dependent change of the kinetic energy (with respect to the sample) into account. In the following, however, the kinetic energy values can be quite close to the vacuum level such that we have to be more carefully with their determination. The values of the kinetic energy indicated in Fig. 7 are those obtained for a Pc coverage of 0.5 ML (with a work function change of about $-0.6 \mathrm{eV}$ ).

Very surprisingly, there is also a lower energy limit for the BP (see full symbols in Fig. 6). For electrons with kinetic energies below about $2 \mathrm{eV}$ the $\mathrm{BP}$ is not present while it is present for higher kinetic energies. We emphasize that a very similar behavior has also been observed with $\mathrm{H}_{2} \mathrm{Pc}$ deposited onto Fe/W(110) (see Sec. II E). This shows that the appearance of a lower energy limit for the BP is not a particularity of the $\mathrm{H}_{2} \mathrm{Pc} / \mathrm{Co}$ system. We note, as we did already for the measurements at higher energies, that the change of the IMFP with energy cannot explain our results for very low kinetic energies. It is known that the IMFP increases dramatically at very low energies with the decrease of energy in Pc films [15]. However, in the energy range around $2 \mathrm{eV}$, where the behavior of the BP changes completely, the IMFP is still too large [15] to explain the quite abrupt change in behavior.

In this context we mention a SPLEEM experiment which some of us performed on another system, namely graphene/Co/Ir(111) [16]. The first point to emphasize is that graphene behaves quite similar to a-C and organic molecules as it exhibits as well the BP, i.e., the presence of a graphene

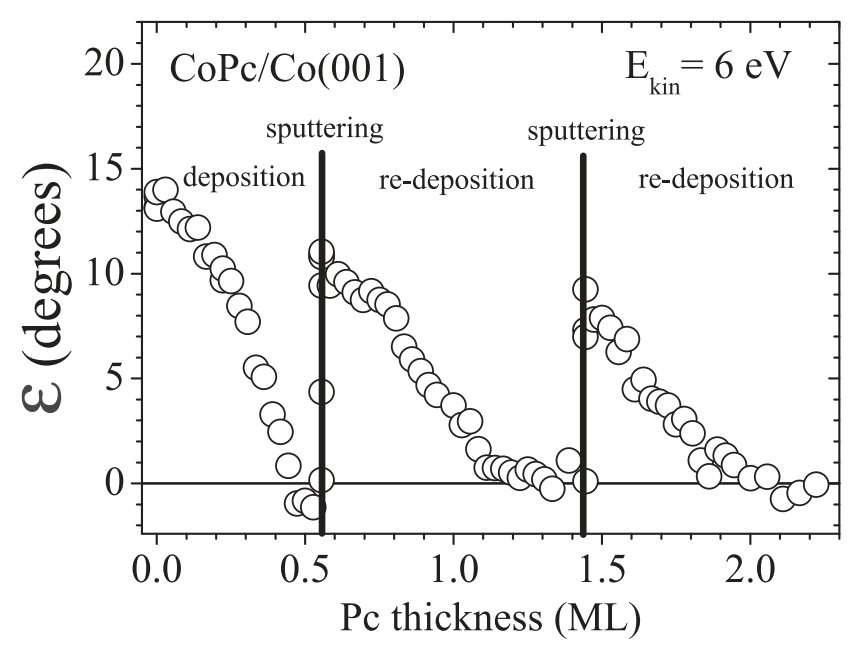

FIG. 8. The precession angle $\varepsilon$ of $\mathrm{Co}$ as a function of $\mathrm{CoPc}$ thickness is shown. The deposition process is interrupted two times by sputtering. The kinetic energy of the incident electrons is $6 \mathrm{eV}$.

layer suppresses the exchange asymmetry almost completely. This is a particularly interesting result, as it shows that the semiconducting character of both a-C and the investigated organic molecules such as Pc is not a necessary condition for the appearance of the BP.

Second and most importantly within our present context, a measurement of $A_{\text {ex }}$ as a function of the electron energy shows a transition from nonbreakdown to breakdown behavior at quite the same kinetic energy of about $2-3 \mathrm{eV}$ as found for $\mathrm{Pc}$ on Co/W(110) and Fe/W(110) (see Fig. 1 (right) in Ref. [16]).

We emphasize that for experimental reasons (mentioned at the beginning of this section) the presence of a lower transition energy of about $2 \mathrm{eV}$ (from breakdown to nonbreakdown behavior) could only be verified in exchange-governed systems (where we measured $A_{\mathrm{ex}}$ in SPLEEM), while the presence of the higher transition energy of about $200 \mathrm{eV}$ could only be verified in spin-orbit governed systems (where we measured $A_{\text {so }}$ with the spin-motion setup). It is thus a priori not excluded that the lower transition energy might exist only for exchange-governed systems, while the higher transition energy might exist only for spin-orbit governed systems.

At the end of this section we are left with the question whether the energies for which the BP disappears correspond to characteristic energy scales of these carbon-based systems.

\section{Does the surface roughness influence the BP?}

In order to see whether the surface roughness has any influence on the BP we performed the following experiment (see Fig. 8). In a first step we deposited CoPc onto a "flat" Co film, i.e., a Co film which has been deposited at room temperature onto a sputtered and annealed $\mathrm{Cu}(001)$ surface, until the breakdown of the $\varepsilon$ signal at about $0.5 \mathrm{ML}$ was completed. In a second step we sputtered [without (!) subsequent annealing and thus without flattening of the Co surface] the CoPc-covered surface slightly until an $\varepsilon$ signal was again measurable. The idea was to introduce a significant roughness into the surface. Then, we started re-deposition of $\mathrm{CoPc}$ and stopped again after completion of the breakdown. 


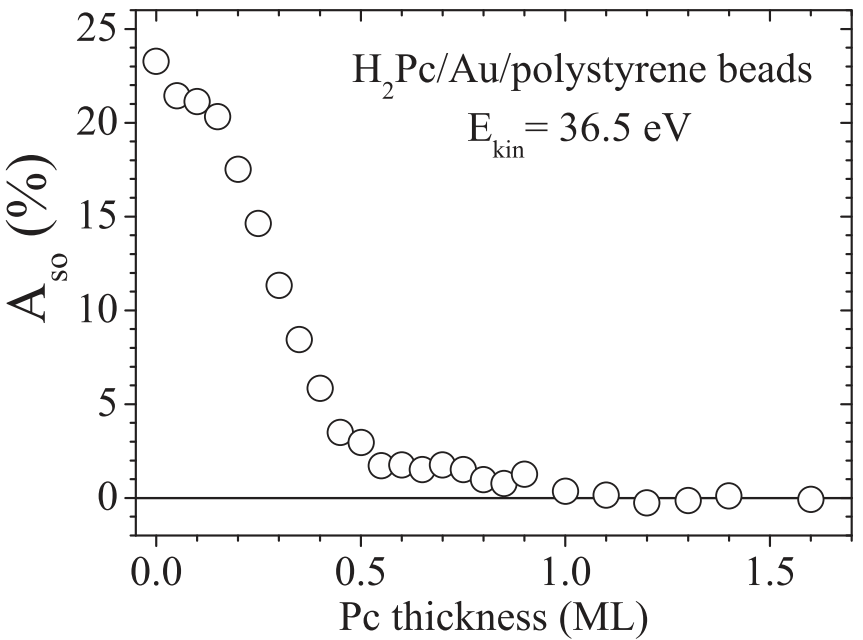

FIG. 9. $A_{\text {so }}$ of Au/polystyrene beads is shown as a function of $\mathrm{H}_{2} \mathrm{Pc}$ thickness. The kinetic energy of the incident electrons is $36.5 \mathrm{eV}$.

In a third step the whole procedure was again repeated. Most importantly, the BP always appears and moreover is always completed at the same thickness of about 0.5 ML whatever the state of the surface is. We note that, on the other hand, the initial $\varepsilon$ signal (before $\mathrm{CoPc}$ deposition) is clearly influenced by this procedure, as it is reduced by $40 \%$ from $14^{\circ}$ to $8^{\circ}$.

In order to have a more defined situation of the surface topography than in the sputtering experiments described above, we designed a particular type of sample. Polystyrene beads with a diameter of $100 \mathrm{~nm}$ have been put onto a Si surface under ambient pressure conditions resulting in an (almost) completely covered Si surface (checked by scanning electron microscopy). In UHV a Au film of $5 \mathrm{~nm}$ thickness has then been deposited on top of the polystyrene beads. This served us as a "rough" substrate for the deposition of $\mathrm{H}_{2} \mathrm{Pc}$. Figure 9, which shows $A_{\mathrm{so}}$ of the Au/polystyrene system as a function of $\mathrm{H}_{2} \mathrm{Pc}$ thickness, proves that the particular surface topography of the sample has no influence on the presence of the BP.

\section{E. Is there percolation?}

The fact that the BP presents very often a threshold thickness led us to the suggestion that the BP might be related to some percolation process, which was also corroborated by kinetic Monte Carlo simulations (see paper I). In order to get more information about this point we performed SPLEEM experiments, as proposed in paper I, to see whether a percolation behavior can be observed within the spatial resolution of $30 \mathrm{~nm}$. Figures 10 and 11 show SPLEEM images for different $\mathrm{H}_{2} \mathrm{Pc}$ coverages on $10 \mathrm{ML} \mathrm{Fe} / \mathrm{W}(110)$ with a kinetic energy of 1.6 and $5.2 \mathrm{eV}$ (with respect to the vacuum level of Fe covered by $0.5 \mathrm{ML} \mathrm{H}_{2} \mathrm{Pc}$ ), respectively. As already seen for $\mathrm{H}_{2} \mathrm{Pc} / \mathrm{Co}$ in Fig. $7 A_{\mathrm{ex}}$ and thus the spin contrast between two oppositely magnetized domains exhibits quite a different behavior as a function of $\mathrm{H}_{2} \mathrm{Pc}$ coverage for kinetic electron energies $E_{\text {kin }}$ below and above the transition energy. While the BP is not present for electrons with $E_{\text {kin }}=1.6 \mathrm{eV}$, it is clearly present for electrons with $E_{\text {kin }}=5.2 \mathrm{eV}$. It has
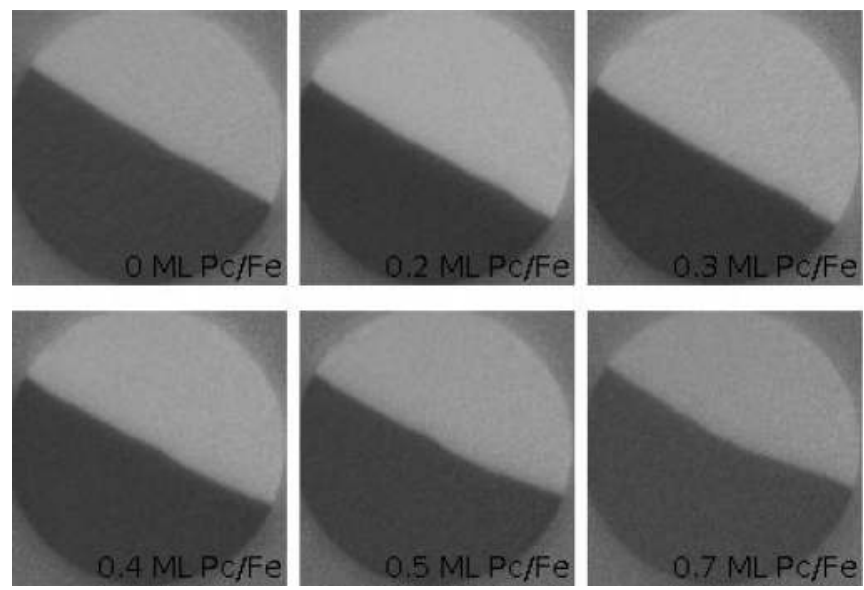

FIG. 10. SPLEEM images showing two in-plane domains as a function of $\mathrm{H}_{2} \mathrm{Pc}$ thickness on $10 \mathrm{ML} \mathrm{Fe} / \mathrm{W}(110)$. The kinetic energy of the electrons with respect to the vacuum level of Fe covered by $0.5 \mathrm{ML} \mathrm{H}_{2} \mathrm{Pc}$ is $1.6 \mathrm{eV}$. The field of view is $6 \mu \mathrm{m}$.

already been discussed in Sec. IIC that the BP obviously disappears for too low kinetic electron energies.

The images for $E_{\text {kin }}=5.2 \mathrm{eV}$ have now been analyzed in more detail. We considered each of the two magnetic domains for the different $\mathrm{H}_{2} \mathrm{Pc}$ coverages and analyzed the respective histograms (of $A_{\mathrm{ex}}$ ). In the case of a percolation process on a length scale larger than the spatial resolution we would expect a speckled appearance of the SPLEEM images in the intermediate $\mathrm{H}_{2} \mathrm{Pc}$ thickness range around 0.2-0.3 ML, i.e., in the thickness range between the onset and the saturation of the BP. This should lead to a two-peaked distribution in the histograms corresponding to the two types of regions in which the BP is either already present or not yet present. However, the analysis of the histograms shows that the distributions can only be described by a single Gaussian line. Figure 12 shows both the average value of the exchange asymmetry $\bar{A}_{\text {ex }}$ and the width $\Delta A_{\mathrm{ex}}$ of the single Gaussian line as a function of $\mathrm{H}_{2} \mathrm{Pc}$ thickness. A thickness-independent linewidth of about $0.4 \%$ is observed over the whole investigated $\mathrm{H}_{2} \mathrm{Pc}$ coverage
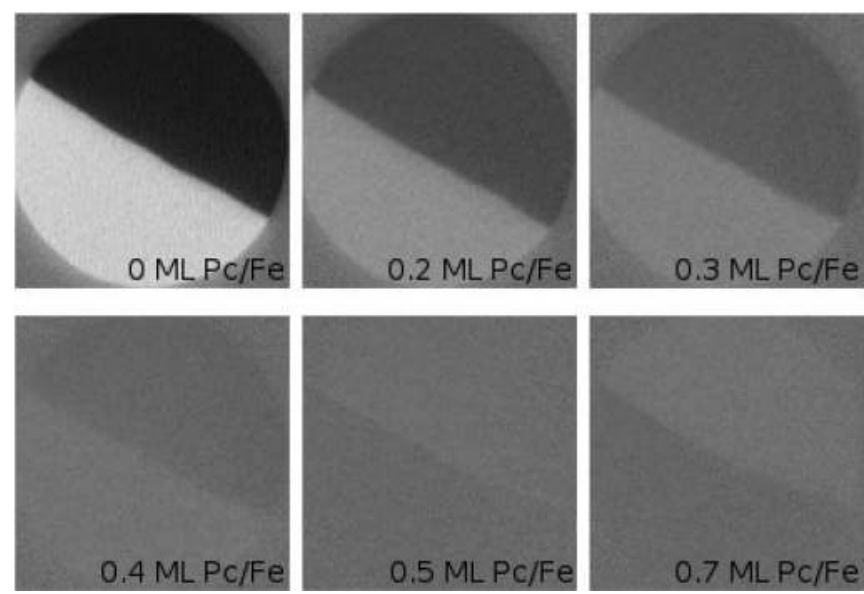

FIG. 11. As in Fig. 10. However, the kinetic energy of the electrons with respect to the vacuum level of Fe covered by $0.5 \mathrm{ML}$ $\mathrm{H}_{2} \mathrm{Pc}$ is $5.2 \mathrm{eV}$. 


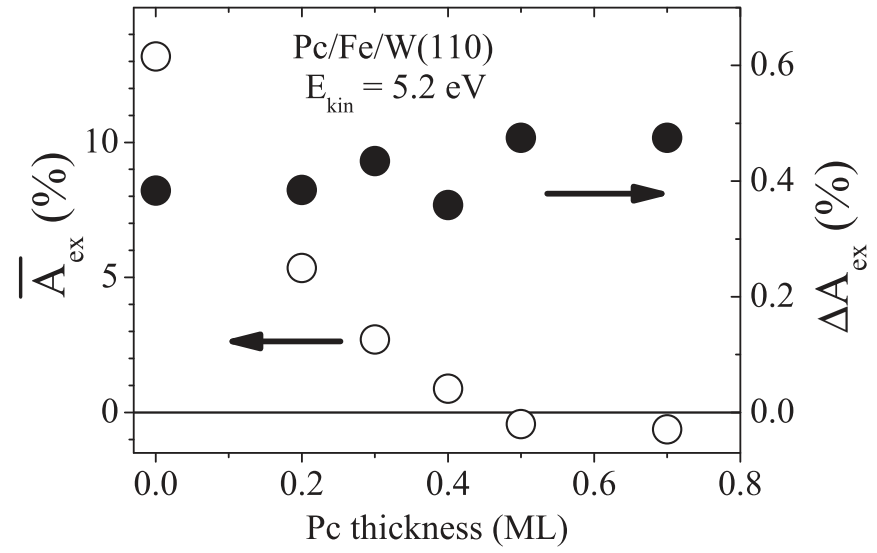

FIG. 12. For the system $\mathrm{H}_{2} \mathrm{Pc} / \mathrm{Fe} / \mathrm{W}(110)$ the average exchange asymmetry $\bar{A}_{\mathrm{ex}}$ and the width $\Delta A_{\mathrm{ex}}$ of the distribution of the histogram as a function of $\mathrm{H}_{2} \mathrm{Pc}$ thickness is shown. The kinetic energy of the electrons is $5.2 \mathrm{eV}$ (see text).

range. On the other hand, in the case of a percolation process on a length scale larger than the spatial resolution of the experiment we would expect two peaks with a separation which is by one order of magnitude larger than the above linewidth. From the above analysis we thus conclude that there is no percolation behavior at least on a length scale larger than the experimental resolution of about $30 \mathrm{~nm}$. However, a percolation behavior on a smaller length scale cannot be excluded and is in fact still suggested by our thickness dependent spin-motion experiments (see paper I).

\section{CONCLUSION}

By performing spin-motion experiments as well as SPLEEM experiments both on spin-orbit and exchange governed metal-organic systems several new aspects of the BP have been studied:

(1) Both spin-dependent reflectivity and spin-dependent reflection phase are subject to the BP in the case of spin-orbit governed metal-organic systems.

(2) The presence of the BP is independent of the angle of incidence of the electrons.

(3) The BP appears in a relatively well defined electron energy range between 2 and $200 \mathrm{eV}$. Outside this range the $\mathrm{BP}$ is not observed.

(4) The presence of the BP does not depend on the roughness of the sample surface.

(5) There is no percolation behavior on a length scale larger than the experimental resolution of about $30 \mathrm{~nm}$. However, a percolation behavior on a smaller length scale cannot be excluded.

An explanation of the BP, however, cannot yet been presented. It is hoped that the experimental results shown in paper I and in the present paper will be sufficiently interesting to motivate theorists to investigate this open problem.

\section{ACKNOWLEDGMENTS}

We acknowledge support from ANR Grant ANR-12-BS1000-401-NANOCELLS. We thank F. Scheurer, M. Alouani, and P. Panissod for discussions on the work.
[1] J. Kirschner, Polarized Electrons at Surfaces, Springer Tracts in Modern Physics, Vol. 106 (Springer, Heidelberg, 1985).

[2] F. Djeghloul, P. Dey, A. Hallal, E. Urbain, S. Mahiddine, M. Gruber, D. Spor, M. Alouani, H. Bulou, F. Scheurer, and W. Weber, Phys. Rev. B 89, 134411 (2014).

[3] F. Djeghloul, F. Ibrahim, M. Cantoni, M. Bowen, L. Joly, S. Boukari, P. Ohresser, F. Bertran, P. Le Fevre, P. Thakur, F. Scheurer, T. Miyamachi, R. Mattana, P. Seneor, A. Jaafar, C. Rinaldi, S. Javaid, J. Arabski, J.-P. Kappler, W. Wulfhekel, N. B. Brookes, R. Bertacco, A. Taleb-Ibrahimi, M. Alouani, E. Beaurepaire, and W. Weber, Sci. Rep. 3, 1272 (2013).

[4] F. Djeghloul, G. Garreau, M. Gruber, L. Joly, S. Boukari, J. Arabski, H. Bulou, F. Scheurer, A. Hallal, F. Bertran, P. Le Fevre, A. Taleb-Ibrahimi, W. Wulfhekel, E. Beaurepaire, S. HajjarGarreau, P. Wetzel, M. Bowen, and W. Weber, Carbon 87, 269 (2015).

[5] P. Dey and W. Weber, J. Phys.: Condens. Matter 23, 473201 (2011).

[6] The normal $\mathbf{n}$ of the reflection plane is given by $\mathbf{n}=\left(\mathbf{k}_{\mathbf{i}} \times \mathbf{k}_{\mathbf{r}}\right)$ / $\left|\mathbf{k}_{\mathbf{i}} \times \mathbf{k}_{\mathbf{r}}\right|$, where $\mathbf{k}_{\mathbf{i}}$ and $\mathbf{k}_{\mathbf{r}}$ are the wave vectors of the incident and the reflected electrons, respectively.

[7] J. Kessler, Polarized Electrons (Springer, Berlin, 1985).

[8] R. Feder, Solid State Commun. 34, 541 (1980).

[9] S. Tanuma, C. J. Powell, and D. R. Penn, Surf. Interface Anal. 21, 165 (1994).
[10] In a SPLEEM instrument spin-polarized electrons, photoemitted from a GaAs-type photocathode, are injected into a spin manipulator where the direction of the spin polarization vector can be oriented in any desired direction. Before being decelerated in the objective lens, the electron beam passes through an illumination column. The electrons arrive at the sample surface under normal incidence. The reflected electrons are then collected in an imaging column and focused on a phosphorous screen, where a magnified image of the sample surface is obtained. The incoming and reflected electron beam are separated in a magnetic beam splitter using the Lorentz force. For more details see N. Rougemaille and A. K. Schmid, Eur. Phys. J. Appl. Phys. 50, 20101 (2010); M. Suzuki et al., Appl. Phys. Express 3, 026601 (2010).

[11] B. G. Johnson, P. J. Berlowitz, D. W. Goodman, and C. H. Bartholomew, Surf. Sci. 217, 13 (1989).

[12] J. G. Ociepa, P. J. Schultz, K. Griffiths, and P. R. Norton, Surf. Sci. 225, 281 (1990).

[13] U. Gradmann and G. Waller, Surf. Sci. 116, 539 (1982).

[14] H. J. Elmers and U. Gradmann, Appl. Phys. A 51, 255 (1990).

[15] S. A. Komolov, E. F. Lazneva, and A. S. Komolov, Tech. Phys. Lett. 29, 974 (2003).

[16] A. D. Vu, J. Coraux, G. Chen, A. T. N'Diaye, A. K. Schmid, and N. Rougemaille, Sci. Rep. 6, 24783 (2016). 